\title{
Sesquiterpenoids of Senecio bonariensis Hook. \& Arn., Asteraceae
}

\author{
Chana de Medeiros da Silva, ${ }^{1}$ Aline Abati Bolzan, ${ }^{1}$ Carlos Augusto Mallmann, ${ }^{2}$ \\ Patrícia Pozzatti, ${ }^{3}$ Sydney Hartz, Alves, ${ }^{3}$ Berta Maria Heinzmann ${ }^{*}, 1,4$
}

\author{
${ }^{1}$ Programa de Pós-graduação em Ciências Farmacêuticas, Centro de Ciências da Saúde, \\ Universidade Federal de Santa Maria, 97105-900 Santa Maria-RS, Brazil \\ ${ }^{2}$ Departamento de Medicina Veterinária Preventiva, Centro de Ciências Rurais, \\ Universidade Federal de Santa Maria, 97105-900 Santa Maria-RS, Brazil \\ ${ }^{3}$ Departamento de Microbiologia, Centro de Ciências da Saúde, Universidade Federal de Santa Maria, \\ 97105-900 Santa Maria-RS, Brazil \\ ${ }^{4}$ Departamento de Farmácia Industrial, Centro de Ciências da Saúde, Universidade Federal de Santa Maria, \\ 97105-900 Santa Maria-RS, Brazil.
}

\begin{abstract}
RESUMO: "Sesquiterpenóides de Senecio bonariensis Hook. \& Arn., Asteraceae". Das folhas de Senecio bonariensis Hook. \& Arn. foram isolados três sesquiterpenóides. $\beta$-Cariofileno (1), óxido de $\beta$-cariofileno (2) e germacreno D (3) foram identificados por CG-IE-EM, IV, ${ }^{1} \mathrm{H}$ and ${ }^{13} \mathrm{C}$ RMN. A importância ecológica e quimiotaxionômica para o gênero Senecio é discutida, assim como a correlação biossintética entre as substâncias isoladas. As substâncias $\mathbf{1}$ e $\mathbf{2}$ tiveram sua atividade antimicrobiana avaliada pelo método de microdiluição em caldo contra cepas bacterianas e fúngicas. Ambas não inibiram o crescimento microbiano nas concentrações testadas.
\end{abstract}

Unitermos: Senecio bonariensis, $\beta$-cariofileno, óxido de $\beta$-cariofileno, germacreno D, quimiotaxonomia, atividade antimicrobiana.

\begin{abstract}
From the leaves of Senecio bonariensis Hook. \& Arn. three sesquiterpenoids were isolated. $\beta$-caryophyllene (1), $\beta$-caryophyllene oxide (2) and germacrene D (3) were characterized by GC-EI-MS, IR, ${ }^{1} \mathrm{H}$ and ${ }^{13} \mathrm{C}-\mathrm{NMR}$ data. Their ecological and chemotaxonomical significance for the genus Senecio are discussed, as well as the biosynthetic correlation between the isolated substances. The antimicrobial activity of substances $\mathbf{1}$ and $\mathbf{2}$ were evaluated by the microdilution method against bacterial and fungal strains. Both compounds did not inhibit the microorganism growth at the tested concentrations.
\end{abstract}

Keywords: Senecio bonariensis, $\beta$-caryophyllene, $\beta$-caryophyllene oxide, germacrene D, chemotaxonom y, antimicrobial activity.

\section{INTRODUCTION}

Species belonging to the family Asteraceae are an important source of terpenes with biological activity (Reina et al., 2001). The genus Senecio (Tribus Senecioneae, Asteraceae) has more than 2000 species. In Brazil, 85 species are been described and form these, 33 occur in southern of the country and 25 were identified in the state of Rio Grande do Sul. Senecio bonariensis Hook. $\&$ Arn. (Asteraceae), popularly known as "margarida-dobanhado-de-buenos aires" is native from South America and can be found in South Brazil, blooming from October to December (Cabrera \& Klein, 1975; Matzenbacher, 1998). This aquatic plant is known for containing furanoeremophilanes (Pomílio \& Jares, 1997; Tettamanzi et al. 1992), steroids (Jares et al., 1990; Tettamanzi et al., 1992) and the pyrrolizidine alkaloids senecionine and platyphylline (Paiva et al., 2004; Silva et al., 2006; Tettamanzi et al., 1992) with toxic properties. This species is used in traditional medicine for the treatment of skin, respiratory and osteoarticular diseases (Bolzan et al., 2007).

In this article we describe the isolation and identification of three compounds from the $\mathrm{CH}_{2} \mathrm{Cl}_{2}$ extract of the aerial parts of $\mathrm{S}$. bonariensis and their antimicrobial evaluation.

\section{MATERIALS AND METHODS}

\section{Plant material}

Leaves of Senecio bonariensis Hook. \& Arn., Asteraceae, were collected in Eldorado do Sul-RS, Brazil, in April 2004 and identified by Prof. Dr. Nelson 
Ivo Matzenbacher. Voucher specimen No SMDB 9519 is preserved in the Herbarium of the Departamento de Botânica, Universidade Federal de Santa Maria, Santa Maria-RS, Brazil.

\section{Extraction and purification}

Fresh leaves of $S$. bonariensis $(2.3 \mathrm{~kg})$ were extracted by maceration with $\mathrm{CH}_{2} \mathrm{Cl}_{2}$, two times. The combined $\mathrm{CH}_{2} \mathrm{Cl}_{2}$ extract was evaporated under vacuum to yield a viscous residue $(13.5 \mathrm{~g})$. The crude extract was fractionated by flash chromatography over silica-gel (Merck, 230-400 mesh), using $\mathrm{CH}_{2} \mathrm{Cl}_{2}$, and $\mathrm{CH}_{2} \mathrm{Cl}_{2}-\mathrm{EtOH}$ mixtures of increasing polarity (stepwise, 100:0 to 80:20), to yield fifteen fractions. The fraction 1 of column 1 (1 g) was chromatographed over $\mathrm{AgNO}_{3}$-impregnated silicagel (Stahl, 1969) eluting with $n$-hexane-acetone (99:1), to yield thirteen new fractions. Fraction 10 of column 2 (253 $\mathrm{mg}$ ) was chromatographed on $\mathrm{AgNO}_{3}$-impregnated silicagel, eluting with hexane-ethyl ether (95:5) to afford 104 $\mathrm{mg}$ of 1 and $16 \mathrm{mg}$ of 2 . Fraction 11 of column 2 (83.5 $\mathrm{mg}$ ) was also chromatographed on $\mathrm{AgNO}_{3}$-impregnated silica-gel, eluting with $n$-hexane:ethyl-ether (98:2) to afford $5 \mathrm{mg}$ of $\mathbf{3}$. The compounds obtained were analyzed by GC-EI-MS, IR, ${ }^{1} \mathrm{H}$ and ${ }^{13} \mathrm{C}$ NMR. Precoated silica gel plates (Merck, Kieselgel 60 F-254, $0.2 \mathrm{~mm}$ ) were used for analytical TLC.

\section{Spectroscopic analysis}

GC-MS were performed using a Hewlett Packard 6890 GC system with a fused capillary column $(30 \mathrm{~m}$ x $0.25 \mathrm{~mm}$ x $0.25 \mu \mathrm{m}, \mathrm{HP}-5 \mathrm{MS}, 5 \%$-phenyl - 95\%methylsiloxane, HP) coupled to Hewlett Packard 5973 mass detector. EI-MS TIC: $70 \mathrm{eV}$; Operating conditions: split injection, split ratio approx. 1:100; temperature programme, $40-260{ }^{\circ} \mathrm{C}$; ramp rate, $4{ }^{\circ} \mathrm{C} / \mathrm{min}$; carrier gas He: flow rate, $1 \mathrm{~mL} / \mathrm{min}$; injector temperature, $220{ }^{\circ} \mathrm{C}$; detector temperature, $220{ }^{\circ} \mathrm{C}$. Data bank NIST, 1998. IR spectra were taken on a Nicolet Magna 550, coupled with HATR for liquid samples and DRIFTS for the solid one. NMR spectra were recorded on a Bruker DPX 300 FT$\mathrm{NMR}$ at $400 \mathrm{MHz}$ for ${ }^{1} \mathrm{H}$ and $100 \mathrm{MHz}$ for ${ }^{13} \mathrm{C}$, in $\mathrm{CDCl}_{3}$ using TMS as internal standard.

$\beta$-Caryophyllene (1): pale yelow oil; $\mathrm{R}_{\mathrm{f}}=0.56\left(\mathrm{AgNO}_{3}-\right.$ impregnated silica-gel $\mathrm{F}_{254}, n$-hexane:ethyl acetate (97:3), three developments, visualization by spraying anisaldehyde- $\mathrm{H}_{2} \mathrm{SO}_{4}$ reagent); EI-MS $m / z 204[\mathrm{M}]^{+}$(4), 189 (10), 175 (5), 161 (18), 147 (15), 133 (50), 120 (24), 105 (39), 93 (65), 91 (66), 79 (60), 69 (63), 55 (35), 41 (100); calcd for $\mathrm{C}_{15} \mathrm{H}_{24} 204 \mathrm{~g}$; $v_{\max } 2930,1450,885 \mathrm{~cm}^{-1}$; ${ }^{1} \mathrm{H}$ NMR: $\delta 5.29$ ( $m, 1 \mathrm{H}$; H-5), 4.82, 4.94 (2 s, 2H; H-15 and $\left.\mathrm{H}-15_{\mathrm{b}}\right), 2.33$ ( $\left.q, J=9.3 \mathrm{~Hz}, 1 \mathrm{H} ; \mathrm{H}-9\right), 2.00-2.20(m$, 4H; H-6 and H-7), 1.91, 1.99 (2 $m, 2 \mathrm{H}$; H-3 and $\mathrm{H}-3$ b), 1.69 ( $m, 1 \mathrm{H} ; \mathrm{H}-1), 1.52,1.67$ ( $m, 2 \mathrm{H} ; \mathrm{H}-10_{\mathrm{a}}$ and $\left.\mathrm{H}-10_{\mathrm{b}}\right)$,
1.61 ( $s, 3 \mathrm{H}$; H-14), 1.26 ( $m, 2 \mathrm{H} ; \mathrm{H}-2), 1.00$ ( $s, 3 \mathrm{H} ; \mathrm{H}-12)$, 0.97 ( $s, 3 \mathrm{H}$; H-13); ${ }^{13} \mathrm{CNMR}: \delta 154.7$ (C-8), 135.5 (C-4), 124.4 (C-5), 111.6 (C-15), 53.7 (C-1), 48.5 (C-9), 40.4 (C-10), 40.0 (C-3), 34.8 (C-7), 33.0 (C-11), 30.1 (C-13), 29.4 (C-6), 28.4 (C-2), 22.6 (C-12), 16.3 (C-14).

Caryophyllene oxide (2): colorless needles; $\mathrm{R}_{\mathrm{f}}=0.67$ $\left(\mathrm{AgNO}_{3}\right.$-impregnated silica-gel $\mathrm{F}_{254}, n$-hexane:ethyl acetate (97:3), three developments, visualization by spraying anisaldehyde- $\mathrm{H}_{2} \mathrm{SO}_{4}$ reagent); EI-MS m/z $220[\mathrm{M}]^{+}$(1), 205 (3), 187 (5), 177 (7), 161 (10), 149 (12), 135 (15), 121 (28), 109 (43), 93 (73), 79 (96), 69 (55), 55 (45), 43 (88), 41 (100); calcd for $\mathrm{C}_{15} \mathrm{H}_{24} \mathrm{O} 220 \mathrm{~g} ; v_{\max } 3070,2950,1620$, 1465, $870 \mathrm{~cm}^{-1}$; ${ }^{1} \mathrm{H}$ NMR: $\delta$ 4.86, $4.98(2 \mathrm{~s}, 2 \mathrm{H}$; H-15 and H-15 $), 2.87$ ( $d d, J=4.6 \mathrm{~Hz}, 1 \mathrm{H} ; \mathrm{H}-5), 2.61$ ( $q, J=9.3$ $\mathrm{Hz}, 1 \mathrm{H}$; H-9), 2.33 (ddd, $J=4.6,7.7$ and $12.4 \mathrm{~Hz}, 1 \mathrm{H}$; H-7a), 2.24 (ddt, $J=4.6,7.7$ and $9.3 \mathrm{~Hz}, 1 \mathrm{H} ; \mathrm{H}_{-6}$ ), 2.11 $\left(m, 2 \mathrm{H} ; \mathrm{H}-3_{\mathrm{b}}\right.$ and $\left.\mathrm{H}-7_{\mathrm{b}}\right), 1.76(t, J=9.3 \mathrm{~Hz}, 1 \mathrm{H} ; \mathrm{H}-1), 1.65$ $\left(m, 3 \mathrm{H} ; \mathrm{H}-2_{\mathrm{b}}\right.$ and $\left.\mathrm{H}-10\right), 1.43(m, 1 \mathrm{H} ; \mathrm{H}-2), 1.30(m, 1 \mathrm{H}$; H-6 ), 1.20 ( $s, 3 \mathrm{H}$; H-14), 1.01 ( $s, 3 \mathrm{H} ; \mathrm{H}-12), 0.98$ ( $m, 1 \mathrm{H}$; H-3 $), 0.98$ ( $s, 3 \mathrm{H}$; H-13); ${ }^{13} \mathrm{CNMR}: \delta 151.9$ (C-8), 112.7 (C-15), 63.7 (C-5), 59.8 (C-4) 50.9 (C-1), 48.7 (C-9), 39.8 (C-10), 39.2 (C-3), 34.0 (C-11), 31.6 (C-6), 30.2 (C-7), 29.9 (C-13), 27.2 (C-2), 21.6 (C-12), 17.0 (C-14).

Germacrene $D$ (3): colorless oil; $\mathrm{R}_{\mathrm{f}}=0.20$ ( $\mathrm{AgNO}_{3}$ impregnated silica-gel $\mathrm{F}_{254}, n$-hexane:ethyl ether (98:2), three developments, visualization by spraying anisaldehyde- $\mathrm{H}_{2} \mathrm{SO}_{4}$ reagent); EI-MS m/z $204[\mathrm{M}]^{+}$(17), 161 (100), 147 (7), 133 (25), 105 (77), 91 (73), 79 (41), 55 (24), 41 (62), calcd for $\mathrm{C}_{15} \mathrm{H}_{24} 204 \mathrm{~g} ; v_{\max } 2930,1450,985$, $880 \mathrm{~cm}^{-1}$; ${ }^{1} \mathrm{H}$ NMR: $\delta 5.78(d, J=15.7 \mathrm{~Hz}, 1 \mathrm{H} ; \mathrm{H}-5), 5.25$ $(d d, J=10$ and $15.9 \mathrm{~Hz}, 1 \mathrm{H}$; H-6), $5.13(d d, J=4.3$ and $10.1 \mathrm{~Hz}, 1 \mathrm{H} ; \mathrm{H}-1), 4.81\left(d, J=2.4 \mathrm{~Hz}, 1 \mathrm{H} ; \mathrm{H}-15_{\mathrm{a}}\right), 4.72(d$, $\left.J=1.7 \mathrm{~Hz}, 1 \mathrm{H} ; \mathrm{H}-15_{\mathrm{b}}\right), 2.40$ (m, 1H; H-3 ), 2.29 ( $m, 1 \mathrm{H}$; H-2 $), 2.22$ ( $m, 2 \mathrm{H}$; H-9), 2.05 ( $\left.m, 1 \mathrm{H} ; \mathrm{H}-3_{\mathrm{b}}\right), 2.01$ ( $m, 1 \mathrm{H}$; $\mathrm{H}-7), 1.98$ ( $m, 1 \mathrm{H} ; \mathrm{H}-2$ ) $), 1.59$ ( $s, 3 \mathrm{H} ; \mathrm{H}-14), 1.48$ ( $m, 2 \mathrm{H}$; $\mathrm{H}-8), 1.43$ ( $m, 1 \mathrm{H}$; H-11), 0.86 ( $d, J=6.6 \mathrm{~Hz}, 3 \mathrm{H}$; H-12), $0.81(d, J=6.6 \mathrm{~Hz}, 3 \mathrm{H} ; \mathrm{H}-13)$.

\section{Antimicrobial activity}

The antimicrobial activity was evaluated as described by Deuschle et al., 2007. The microorganisms tested were Saphylococcus aureus ATCC 25923, Saccharomyces cerevisiae ATCC 2601, Pseudomonas aeruginosa ATCC 27853, Candida albicans ATCC 90028, Acinetobacter baumannii and Prototheca zopfii (clinical isolates). Additionally, compound $\mathbf{1}$ was tested against Candida tropicalis ATCC 750 and compound 2 was evaluated against Escherichia coli ATCC 25922 and Klebsiella pneumoniae ATCC 10031. For compound 1 the tested concentrations ranged from 0.025 and $5 \mathrm{mg} / \mathrm{mL}$ while for compound 2 they varied between 53.62 and 1716 $\mu \mathrm{g} / \mathrm{mL}$. 


\section{RESULTS AND DISCUSSION}

The structure of $\mathbf{1}$ was established as $\beta$-caryophyllene, a natural product isolated from several other Senecio species (Bohlmann \& Zdero, 1982a; Bohlmann et al., 1981, 1986; Jakupovic et al., 1991; Kite \& Smith, 1997; Mericli et al., 1989; Urzúa \& Andrade, 2001). This compound displays antiinflamatory (Tambe et al., 1996), anticarcinogenic (Zheng et al., 1992) and cytotoxic (Kubo et al., 1996) activities. Besides, $\beta$-caryophyllene can autoxidize when air exposed to produce $\beta$-caryophyllene oxide, a compound with moderate allergenic activity (Sköld et al., 2006).

Compound 2 was proved to be $\beta$-caryophyllene oxide, which was also described for some Senecio species (Bohlmann et al., 1986; Jakupovic et al., 1991; Reina et al., 2002). This compound displays citotoxic (Kubo et al., 1996; Reina et al., 2002; Sibanda et al., 2004), antimalarial (Thebtaranonth et al., 1995), anticarcinogenic (Zheng et al., 1992), repellent against the mosquito Anopheles gambiae (Omolo et al., 2004), as well as antifungal properties (Yang et al., 2000). Although this substance is an artefact, it has been approved by the FDA as a food and cosmetic preservative and has been included by the European Council in the list of natural and synthetic flavouring substances (FDA, 1973). It appears to be tolerable, safe and toxic-free at the usual concentrations (Yang et al., 2000).

The structure of $\mathbf{3}$ was established as germacrene $\mathrm{D}$, a hydrocarbon commonly found as plant constituent and considered to be a key intermediate in the biosynthesis of many sesquiterpenoids (Bülow \& König, 2000; Prosser et al., 2004; Steliopoulos et al., 2002). This compound is present in several plant families, including Asteraceae (Mouzuraitis et al., 2002) and is frequently found in Senecio species (Bohlmann \& Zdero, 1982b; Bohlmann et al., 1980, 1983, 1985; Deuschle, 2003; Deuschle et al., 2007; Francescato et al., 2003; Francescato, 2007; Murari 2007; Jakupovic et al., 1991). As biological activity is to emphasize it's ability to interact with insects and other organisms (Mozuraitis et al., 2002; Rostelien et al., 2000; Stranden et al., 2002). According to literature data, the content of germacrene $\mathrm{D}$ varies among plant species from pure (+)- or (-)-enantiomers to the racemate, controlled by separate synthases (Schmidt et al., 1998). Although this compound is present as its (-)-enantiomer in most higher plants, for several the occurrence of both enantiomers has been described (Bülow \& König, 2000). Once the gas chromatography employed wasn't enantioselective and the optical rotation of compound 3 has not been measured, it remains unknown which enantiomer is present in $S$. bonariensis.

The three compounds have a relative close biosynthetic correlation as shown in Figure 1. From trans,trans-FPP, several enzymatic reactions occur, conducting to cyclizations, eliminations and subsequent rearrangements, originating several intermediates, as the germacrenyl- and humulyl-cations, which are responsible for the formation of different sesquiterpenoids, such as germacrene $\mathrm{D}$ and $\beta$-caryophyllene. The latter substance, through a process of autoxidation and degradation, following by cyclization and epoxidation, produces $\beta$-caryophyllene oxide.

The large genus Senecio is chemically and morphologically not very homogenous (Dupré et al., 1991), and there are still problems concerning the delimitation of species groups within the genus (Bohlmann et al., 1980). Thus, terpenoidic quinones and triterpenes are widespread in some species, while sesquiterpenes are very localized and poorly represented (Tundis et al., 2005). Otherwise, there are differences between succulent Senecio species and the other ones (Bohlmann et al., 1980, 1981). In our systematic studies with Brazilian indigenous Senecio species, we observed that sesquiterpenes are widespread among the analyzed plants. It seems that the most South-Brazilian Senecio species are rich in caryophyllane, germacrane-, eudesmane- and gauaiane-derivatives (Deuschle et al., 2007; Francescatto et al., 2003; Murari, 2007; Ghisalberti, 1994), which are formed form trans,trans-FPP (Bülow \& König, 2000). However, the succulent species contain mainly bisabolane derivatives (Rücker et al., 1996, 1997), which are originated from cis, trans-FPP (Thol, 2006). These findings could be of chemotaxonomical significance and may be contribute to the botanical classification of these plants.

Compound $\mathbf{3}$ has its antimicrobial activity evaluated by an earlier work (Deuschle et al., 2007). Although the isolated sesquiterpernoids are often find in essential oils which presented antimicrobial activity, they were inactive at the tested concentrations.
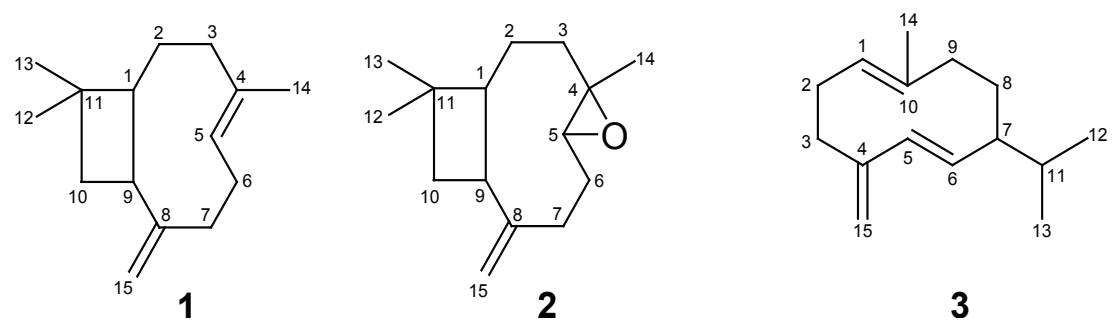

3 


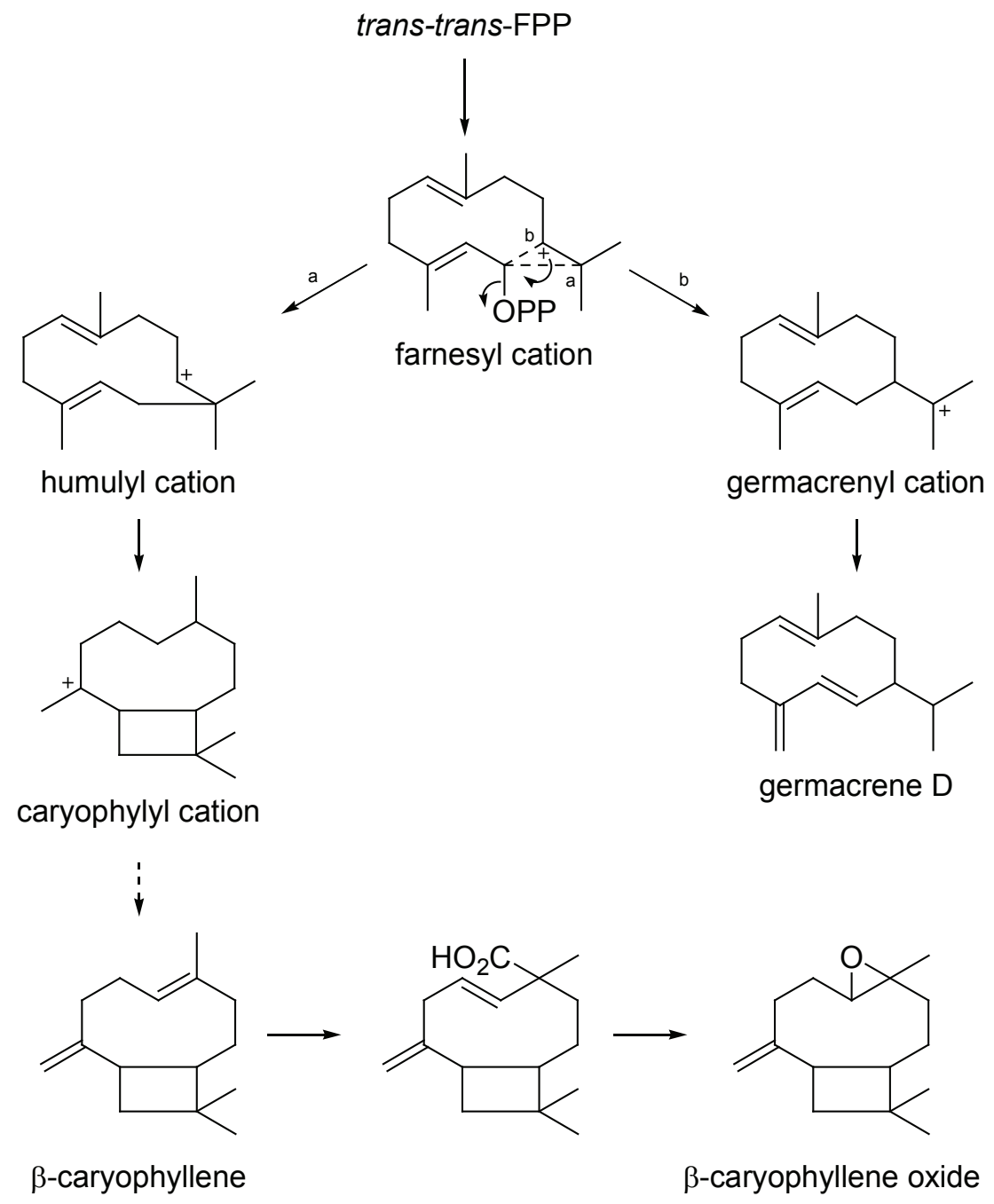

Figure 1. Biosynthesis of germacrene D and $\beta$-caryophyllene, as well as formation of caryophyllene oxide (adapted from Bülow \& König, 2000; Fricke, 1999; Greenhagen, 2003; Prosser et al., 2002).

\section{ACKNOWLEDGEMENTS}

We thank to Nelson Ivo Matzenbacher (Programa de Pós-Graduação em Botânica/UFRGS) for locating and identified plant material. This work was supported by CNPq and CAPES.

\section{REFERENCES}

Bohlmann F, Ziesche J, King RM, Robinson H 1980. Seven furanoeremophilanes from three Senecio species. Phytochemistry 19: 2675-2679.

Bohlmann F, Zdero C, King M, Robinson H 1981. The first acetylenic monoterpene and other constituents from Senecio clevelandii. Phytochemistry 20: 2425-2427.

Bohlmann F, Zdero C 1982a. Sandaracopimarene derivatives from Senecio subrubriflorus. Phytochemistry 21: 16971700.
Bohlmann F, Zdero C 1982b. Germacrene derivatives and other sesquiterpenes from Senecio species and Lordhowea insularis. Phytochemistry 21: 2537-2541.

Bohlmann F, Nezhun A, King R, Robinson H 1983. Two sesquiterpenes from Senecio species. Phytochemistry 22: 1675-1677.

Bohlmann F, Zdero C, Jakupovic J, Misra L, Banerjee S, Singh P, Baruah R, Metwally M, Schmeda-Hirschmann G, Vincent L, King R, Robinson H 1985. Eremophilane derivatives and other constituents from Senecio species. Phytochemistry 24: 1249-1261.

Bohlmann F, Zdero C, Jakupovic J, Grenz M, Castroa V, King RM, Robinson H, Vincent L 1986. Further pyrrolizidine alkaloids and furoeremophilanes from Senecio species. Phytochemistry 25: 1151-1159.

Bolzan AA, Silva CM, Francescato LN, Murari AL, Silva GNS, Heldwein CG, Heinzmann B 2007. Espécies de Senecio na medicina popular da América Latina e toxicidade 
relacionada a sua utilização. Latin Am J Pharm 26: 619625.

Bülow N, König, WA 2000. The role of germacrene D as a precursor in sesquiterpene biosynthesis: investigations of acid catalyzed, photochemically and thermally induced rearrangements. Phytochemistry 55: 141-168.

Cabrera AL, Klein RM 1975. Compostas. In: Reitz PR (Eds): Flora Ilustrada Catarinense. Herbário Barbosa Rodrigues, Itajaí, Santa Catarina, Brazil.

Deuschle RAN 2003. Atividade antimicrobiana e análise fitoquímica de Senecio desiderabilis Vellozo (Asteraceae). Santa Maria, 124f. Dissertação de Mestrado - Programa de Pós-graduação em Ciências Farmacêuticas, Universidade Federal de Santa Maria.

Deuschle RAN, Camargo T, Alves SH, Mallmann CA, Heinzmann BM 2007. Fracionamento do extrato diclorometânico de Senecio desiderabilis Vellozo e avaliação da atividade antimicrobiana. Rev Bras Farmacogn 17: 71-75.

Dupré S, Grenz M, Jakupovic J, Bohlmann F, Niemeyer HM 1991. Eremophilane, germacrane and shikimic acid derivatives from Chilean Senecio species. Phytochemistry 30: 12111220.

Food and Drug Administration 1973. Washington, DC, USA. Rules and Regulations: title 21 - Food and drugs, food additives, synthetic flavoring substances and adjuvants Fed Regist, 95 (38), 12913-12914.

Francescato LN, Alves SH, Nahrstedt A, Heinzmann BM 2003. Partial synthesis of Germacrene D-1-hydroperoxide. Braz J Pharm Sci 39: 226.

Francescato LN 2007. Constituintes de Senecio heterotrichius DC.: isolamento, elucidação estrutural, derivatização e avaliação da atividade antimicrobiana; estudo dos constituintes voláteis de outras espécies de Senecio. Santa Maria, 158f. Dissertação de Mestrado - Programa de Pósgraduação em Ciências Farmacêuticas, Universidade Federal de Santa Maria.

Fricke C 1999. Terpenoide Inhaltsstoffe von Lebermoosen und Heilpflanzen. Germany, 131p. PhD thesis, University of Hamburg.

Ghisalberti EL 1994. The daucane (carotane) class of sesquiterpenes. Phytochemistry 37: 597-623.

Greenhagen BT 2003. Origins of isoprenoid diversity: a study of structure-function relationships in sesquiterpene synthases. 145p. Tese de Doutorado em Filosofia, University of Kentucky.

Jakupovic J, Grenz M, Bohlmann F, Niemeyer H 1991. Furoeremophilanes and other constituents from Chilean Senecio species. Phytochemistry 30: 2691-2693.

Jares EA, Tettamanzi, MC, Pomilio AB 1990. Sitosterol 3-O$\beta$-D-glucuronopyranoside from Senecio bonariensis. Phytochemistry 29: 340-341.

Kite GC, Smith SAL 1997. Inflorescence odour of Senecio articulatus: temporal variation isovaleric acid levels. Phytochemistry 45: 1135-1138.

Kubo I, Chaudhuri SK, Kubo Y, Sanchez Y, Ogura T, Saito T, Ishikawa H, Haraguchi H 1996. Cytotoxic and oxidative sesquiterpenoids from Heterotheca inuloides. Planta Med 62: 427-430.

Matzenbacher NI 1998. Ocomplexo "Senecionoide" (AsteraceaeSenecioneae) no Rio Grande do Sul Brasil. Porto Alegre, 274p. Tese de Doutorado, Programa de pós-graduação em Botânica, Universidade Federal do Rio Grande do Sul.

Mericli AH, Mericli F, Jakupovic J, Bohlmann F, Dominguez XA, Vega HS 1989. Eremophilane derivatives and other constituents from Mexican Senecio species. Phytochemistry 28: 1149-1153.

Mozuraitis R, Stranden M, Ramirez MI, Borg-Karlson AK, Mustaparta H 2002. (-) - Germacrene D increases attraction and oviposition by the tobacco budworm moth Heliothis virescens. Chem Senses 27: 505-509.

MurariAL2007. Constituintes voláteis e atividade antimicrobiana de Senecio crassiflorus var. crassiflorus. Santa Maria, 133f. Dissertação de Mestrado - Programa de Pósgraduação em Ciências Farmacêuticas, Universidade Federal de Santa Maria.

Omolo MO, Okinyo D, Ndiege IO, Lwande W, Hassanali A 2004. Repellency of essential oils of some Kenyan plants against Anopheles gambiae. Phytochemistry 65: 27972802.

Paiva JA, Barata LES, Trigo JR 2004. Pyrrolizidine alkaloids in three Senecio species from southern Brazil. Biochem Syst Ecol 32: 1219-1222.

Pomilio AB, Jarres EA 1997. Toxic furanoeremophilanes from Senecio bonariensis. Int J Pharmacogn 35: 207-211.

Prosser I, Phillips AL, Gittings S, Lewis MJ, Hooper AM, Pickett JA, Beale MH 2002. (+) - (10R) - Germacrene A synthase from goldenrod, Solidago canadensis; cDNA isolation, bacterial expression and functional analysis. Phytochemistry 60: 691-702.

Prosser I, Altug IG, Phillips AL, König WA, Bouwmeester HJ, Beale MH 2004. Enantiospecific (+) and (-) Germacrene D synthases, cloneed from goldenrod, reveal a functionally active variant of the universal isoprenoidbiosynthesis aspartate-rich motif. Arch Biochem Biophys 432: 136-144.

Reina M, Gonzalez-Coloma A, Gutierrez C, Cabrera R, Rodriguez ML, Fajardo V, Villarroel L 2001. Defensive chemistry of Senecio miser. J Nat Prod 64: 6-11.

Reina M, Nold M, Santana O, Orihuela JC, González-Coloma A 2002. C-5-Substituted antifeedant silphinene sesquiterpenes from Senecio palmensis. J Nat Prod 65: 448-453.

Rostelien T, Borg-Karlson AK, Fäldt J, Jacobsson U, Mustaparta H 2000. The plant sesquiterpene germacrene D specifically actives a major type of antennal receptor neuron of the tobacco budworm moth Heliothis virescens. Chem Senses 25: 141-148.

Rücker G, Schenkel EP, Manns D, Mayer R, Heiden K, Heinzmann BM 1996. Sesquiterpene peroxides from Senecio selloi and Eupatorium rufescens. Planta Med 62: 565-566.

Rücker G, Breitmaier E, Manns D, Maier W, Marek A, Heinzmann 
B, Heiden K, Seggewies S 1997. Antimalarial activity of 1,4-Epidioxy-bisabola-2,12-diene derivatives. Arch Pharm Pharm Med Chem 330: 12-16.

Schmidt CO, Bouwmeester HJ, Dekraker JW, König WA 1998. Biosynthesis of (+)- and (-) Germacrene D in Solidago canadensis: isolation, and characterization of two enantioselective germacrene D synthases. Angew Chem Int Ed 37: 1400-1402.

Sibanda S, Chigwada G, Poole M, Gwebu ET, Noletto JA, Schmidt JM, Rea AI, Setzer WN 2004. Composition and bioactivity of the leaf essential oil of Heteropyxis dehniae from Zimbabwe. J Ethnopharmacol 92: 107-111.

Silva CM, Bolzan AA, Heinzmann BM 2006. Alcalóides pirrolizidínicos em espécies do gênero Senecio. Quím Nova 29: 1047-1053.

Sköld M, Karlberg A, T Matura, M Börje A 2006. The fragrance chemical $\beta$-caryophyllene - air oxidation and skin sensitization. Food Chem Toxicol 44: 538-545.

Stahl E 1969. Thin-layer chromatography - a laboratory handbook, second ed. Berlin: Springer.

Steliopoulos P, Wust M, Adamb K, Mosandla A 2002. Biosynthesis of the sesquiterpene germacrene $\mathrm{D}$ in Solidago canadensis: ${ }^{13} \mathrm{C}$ and ${ }^{2} \mathrm{H}$ labeling studies. Phytochemistry 60: 13-20.

Stranden M, Borg-Karlson AK, Mustaparta H 2002. Receptor neuron discrimination of the germacrene $\mathrm{D}$ enantiomers in the moth Helicoverpa armigera. Chem Senses 27: 143-152.

Tambe Y, Tsujiuchi H, Honda G, Ikeshiro Y, Tanaka S 1996. Gastric cytoprotection of the non-steroidal antiinflamatory sesquiterpene, $\beta$-caryophillene. Planta Med 62: 469-470.

Tettamanzi MC, Jares EA, Pomilio AB 1992. Constituents of Senecio bonariensis roots. Fitoterapia 63: 551-552.

Thebtaranonth C, Thebtaranonth Y, Wanauppathamkul S, Yuthavong Y 1995. Antimalarial sesquiterpenes from tubers of Cyperus rotundus: structure of 10,12peroxycalamenene, a sesquiterpene endoperoxide. Phytochemistry 40: 125-128.

Thol D 2006. Terpene synthases and the regulation, diversity and biological roles of terpene metabolism. Curr Opinion Plant Biol 9: 297-304.

Tundis R, Passalacqua NG, Peruzzi L, Statti GA, Bonesi M, Loizzo MR, Conforti F, Cesca G, Menichini F 2005. Comparative chemical variability of the non-polar extracts from Senecio cineraria group (Asteraceae). Biochem Syst Ecol 33: 1071-1076.

Urzúa A, Andrade L 2001. Comparative chemical composition of the resinous exsudates from Senecio adenotrichius and $S$ viscosissimus. Biochem Syst Ecol 29: 865-867.

Yang D, Michel L, Chaumont JP, Millet-Clerc J 2000. Use of caryophyllene oxide as an antifungal agent in an in vitro experimental model of onychomycosis. Mycopathol 148: 79-82.

Zheng GQ, Kenney PM, Lam LKT 1992. Sesquiterpenes from clove (Eugenia caryophyllata) as potential anticarcinogenic agents. J Nat Prod 55: 999-1003. 